Basic Health Sciences

Poster

Abstract ID: 23

\title{
Nigella sativa oil extracts improves dyslipidaemia in high fat diet (HFD) animal model
}

Roslina Abdul Rahim ${ }^{\mathrm{a}}$ | Muhammad Taufiq Badaruddin ${ }^{\mathrm{a}}$ | Qamar Uddin Ahmed ${ }^{\mathrm{b}}$ | Siti Aesah @ Naznin Muhammad ${ }^{\mathrm{a}}$

${ }^{a}$ Kulliyyah of Medicine, International Islamic University Malaysia

${ }^{b}$ Kulliyyah of Pharmacy, International Islamic University Malaysia

Introduction: Nigella sativa has been reported to have anti-inflammatory and antioxidative properties. We aimed to investigate the effects of Nigella sativa oil extract on the high fat diet (HFD) fed animal model. Methods: Thirty male Sprague-Dawley rats were used. Four groups $(n=7)$ were fed with high fat diet (HFD) rat pellet orally for 10 weeks. The rats with HFD groups were subsequently randomly divided again into 4 groups. One group was continued with HFD while the other 3 groups were continued with the HFD in addition to Nigella sativa oil extract treatment at three different concentrations $(0.5,1$ and $1.5 \mathrm{ml} / \mathrm{kg} /$ day) for another 4 weeks. Blood biochemical analysis and histological assessment of liver were subsequently performed. Results: Animals fed with HFD had increased aspartate aminotransferase (AST), alkaline phosphatase (ALP), albumin, cholesterol/HDL ratio, triglyceride (TG) levels, but decreased globulin, albumin/globulin ratio and high density lipoprotein (HDL) levels. Meanwhile, HFD animals treated with Nigella sativa oil extract showed a significant increase in the globulin and HDL levels, but reduced ALP, albumin, cholesterol/HDL ratio and TG levels. Nigella sativa oil extract at the higher doses displayed much better effect. Histologically the liver in all groups exhibited micro vesicular steatosis. Conclusions: Nigella sativa oil extracts improve the dyslipidaemia in animal models with HFD.

KEYWORDS: Nigella sativa, high fat diet, dyslipidaemia 